\title{
Key membership and implied harmony in Western tonal music: Developmental perspectives
}

\author{
LAUREL J. TRAINOR \\ McMaster University, Hamilton, Ontario, Canada \\ and \\ SANDRA E. TREHUB \\ University of Toronto, Toronto, Ontario, Canada
}

\begin{abstract}
We investigated the role of key membership and implied harmony in adults' and children's perception of tone sequences. Listeners were evaluated on their ability to detect three types of changes in one note of a well-structured Western tonal melody. In one change (out-of-key) the new note was not in the basis key, in another (out-of-harmony) it was in the key but not in the implied harmony, and in the third (within-harmony) it was in both the key and the implied harmony. Adults and 7-year-olds performed better on the out-of-key and out-of-harmony changes than on the within-harmony change, reflecting their implicit knowledge of key membership and implied harmony. Five-year-olds performed better on the out-of-key change than on the other two changes, reflecting the influence of key membership but not implied harmony. We consider the developmental precedence of key membership over implied harmony in the context of cross-cultural and theoretical perspectives.
\end{abstract}

Western listeners, including those who are musically untrained, process melodies according to music-theoretic rules of tonality (e.g., Cuddy, 1991; Cuddy, Cohen, \& Miller, 1979; Deutsch \& Feroe, 1981; Dowling \& Harwood, 1986; Howell, Cross, \& West, 1985; Krumhansl, 1990; Pick, 1979; Shepard, 1982; Sloboda, 1985; Thompson, 1993), as described in standard texts of music theory (e.g., Aldwell \& Schachter, 1989). One example of the convergence of perceptual processes and music-theoretic rules concerns key relations in music. Western tonal compositions are said to be in a particular key (e.g., C major), which means that the component notes of a composition belong to the scale bearing that name (i.e., $\mathrm{C}$ major scale), with specific notes serving different functions. Perceptual studies reveal that adults more readily detect changes that introduce a nonkey note than changes that do not (e.g., Cuddy et al., 1979; Trainor \& Trehub, 1992). Western music theory also defines some keys as being closely related (e.g., $\mathrm{C}$ major and $\mathrm{G}$ major) and others as distantly related (e.g., C major and F\# major). Again, there are psychological consequences of such key relations; adults' and children's perceptions of pairs of melodies are influenced by the relatedness of the melodies' keys (Bartlett \& Dowling, 1980; Takeuchi \& Hulse, 1992; Trainor \& Trehub, 1993a; Trehub, Morrongiello, \& Thorpe,

This research was supported by grants from the Natural Sciences and Engineering Research Council of Canada. We are grateful to E. Glenn Schellenberg, Ron Racine, and John Platt for their critical reviews of the manuscript. Send reprint requests to L. J. Trainor, Department of Psychology, McMaster University, Hamilton, Ontario, Canada L8S 4K1.
1985). Nevertheless, the extent to which the convergence of music theory and perception derives from nature or nurture is unknown.

The study of musical development provides a means of assessing the relative contributions of perceptual predispositions and exposure. Features of music with an innate basis are more likely to be perceived and produced earlier than those that are acquired. Indeed, there has been significant progress in identifying perceptual processing biases in infancy (see Trehub \& Trainor, 1990, 1993; Trehub, Trainor, \& Unyk, 1993; Trehub \& Unyk, 1991). Developmental music research can also provide a window on the relative ease or difficulty of processing different structural features of music. For features that must be learned, those acquired earlier must be intrinsically easier to learn than those acquired later, given comparable exposure. In the present investigation, we examine two features of Western tonal music in developmental perspective: key membership and implied harmonic function.

Pitch structure in Western tonal music has two aspects: melody, or successive pitch structure, and harmony, or simultaneous pitch structure. Melody and harmony are not independent, however, in that every melody is considered to embody an implied harmony (see, e.g., Bharucha, 1984). In other words, specific melodic structures are thought to dictate appropriate harmonic accompaniment. In fact, definitions of melody often refer specifically to harmonic implications. According to Terhardt, "What makes a tone sequence a melody is harmonic and rhythmic organization" (1991, p. 217). Empirical research reveals, moreover, that Western adults with no mu- 
sical training are sensitive to aspects of implied harmony (Platt \& Racine, 1994), although melody and harmony seem to contribute somewhat independent information about key structure (Thompson \& Cuddy, 1989, 1992).

Cross-cultural examination reveals that melody is found in virtually all musical systems, but that harmony may be unique to Western music (Dowling \& Harwood, 1986). Virtually all musical systems base melodic creation on scales, which greatly restricts the choice of component notes. Different musical systems use different scales, making it necessary for listeners to become familiar, at least implicitly, with the particular scales of their musical culture. Therefore, the acquisition of implicit knowledge about key membership (i.e., which notes belong to a particular scale or key) can be considered a universal of musical development. Presumably, musical systems have developed in accordance with the human auditory system, at least to some extent. Universal aspects of music are likely to be more perceptually salient than nonuniversal aspects-the latter are likely to require greater exposure for comparable ease of processing (Trehub \& Trainor, 1990, 1993; Trehub et al., 1993). In this light, one would expect the perception of melody to be more fundamental than the perception of harmony, and one would also expect it to develop earlier, even for listeners exposed to both kinds of information.

The search for musical universals has met with limited success, yielding very few and very basic cross-cultural similarities (Harwood, 1976). Nevertheless, these similarities may guide the identification of elementary or readily acquired aspects of musical processing. In most musical systems, pitch is related to fundamental frequency on a logarithmic scale, which means that the distance between two pitches is directly related to the ratio of their fundamental frequencies. Tones that are one octave apart (i.e., fundamental frequencies standing in a $1: 2$ ratio) sound very similar in musical contexts, are functionally equivalent, and are often assigned the same note name. Moreover, research with very young infants indicates that even they perceive the similarity of tones that are one octave apart (Demany \& Armand, 1984). Another near-universal is the use of a small set of discrete pitches per octave (scales) and their repetition in successive octaves. A culture-specific example is the Western major scale, which is composed of tones separated by two, two, one, two, two, two, and one semitones, where a semitone is one twelfth of an octave (on a $\log _{2}$ scale). The first note, or tonic, is considered the most important, and compositions based on a particular scale are said to be in the key of the tonic. The notes C, D, E, F, G, A, and B $(262,293$, $329,349,391,440$, and $492 \mathrm{~Hz}$, starting on middle C) form a major scale; compositions based on this scale would be in the key of $\mathrm{C}$ major. The second most important note is the fifth, or dominant, which is $\mathrm{G}$ in the case of the C major scale.

Western harmonic structure is based on chords (i.e., three or more simultaneous tones), which can be constructed on each note of the scale. The two most impor- tant chords are based on the tonic and dominant notes, designated the tonic chord ( $\mathrm{C} \mathrm{E} \mathrm{G}$ in the key of $\mathrm{C}$ major) and dominant chord (G B D in the key of $C$ major), respectively. A variant of the dominant chord is the dominant seventh chord (G B D F in the key of C major). For Western listeners, at least, dominant harmony is thought to produce tension, which is resolved when followed by tonic harmony. It is not surprising, then, that Western compositions typically begin and end with tonic harmony.

Recent research has revealed that infants are similar to adults in their perception of a number of aspects of musical structure. For example, infants and adults recognize melodies presented in transposition (i.e., at different starting pitches), indicating that they give precedence to relative pitch information (i.e., the pitch distance, or interval, between notes) over absolute pitch (e.g., Cohen, Thorpe, \& Trehub, 1987; Trainor \& Trehub, 1992, 1993a). Infants, like adults, are sensitive to pitch contour (the up/ down/same pattern of pitch changes), readily detecting any melodic changes that alter the contour (Trehub, Bull, \& Thorpe, 1984; Trehub, Thorpe, \& Morrongiello, 1985, 1987). Moreover, infants and adults exhibit enhanced processing for some melodies (Cohen et al., 1987; Trainor \& Trehub, 1993a, 1993b; Trehub, Thorpe, \& Trainor, 1990 ), although some of the factors underlying the ease or difficulty of melodic discriminations remain unclear. Both infants and adults, however, find it easier to discriminate changes to melodies if they contain perfect fifth intervals (i.e., notes standing in a 2:3 frequency ratio; see Cohen et al., 1987; Trainor \& Trehub, 1993b; Trehub et al., 1990). It is also easier to discriminate differences between transposed melodies if the melodies are related by a perfect fifth (Trainor \& Trehub, 1993a).

Infants differ from adults in other aspects of musical processing, such as their implicit knowledge of key membership. Trainor and Trehub (1992) compared infants' and adults' ability to detect two types of changes to a single note of a well-structured Western melody $\left(\mathrm{C}_{4}, \mathrm{E}_{4}, \mathrm{G}_{4}\right.$, $F_{4}, D_{4}, G_{3}, C_{4}, E_{4}, D_{4}, C_{4}$-the subscript represents the octave from which the note is drawn, where $\mathrm{C}_{4}$ is middle C: $262,329,391,349,293,195,262,329,293$, and $262 \mathrm{~Hz}$ in the key of C major). One of these involved the alteration of a single note so that it was outside the key of the melody (in the key of C major, $\mathrm{G}_{3}$, or $195 \mathrm{~Hz}$, was changed to $A b_{3}$, or $207 \mathrm{~Hz}$ ); the other involved a singlenote change that remained within the key of the melody $\left(G_{3}\right.$, or $195 \mathrm{~Hz}$, was changed to $B_{3}$, or $\left.246 \mathrm{~Hz}\right)$. Not surprisingly, the adults found the within-key change more difficult to detect than the out-of-key change; the latter change created an atypical Western melody. By contrast, the infants performed equivalently on both changes, actually outperforming the adults on the within-key change. These findings imply that infants are unaffected by key membership, lacking knowledge about the underlying scale structure of Western music. Research with foreign musical materials (Lynch, Eilers, Oller, \& Urbano, 1990; Lynch, Eilers, Oller, Urbano, \& Wilson, 1992) indicates that mu- 
sically untrained adults more readily detect changes in the familiar Western major scale than in the unfamiliar Javanese pelog scale, but that infants perform equivalently on both tasks. Infants' nondifferential performance on native and foreign scales implies that the Western major scale does not have preferential status, at least for 6-monthold listeners.

There are relatively few studies of preschool and schoolage children's processing of melodic pitch structure. Nevertheless, untrained Western children 10-13 years of age are better able to detect changes in the Western major scale than in the Javanese pelog scale (Lynch \& Eilers, 1991). There are suggestions that, even by age 6 , children find that changes that go outside the key of a wellknown melody are easier to detect than those that remain within the key (Trehub, Morrongiello, \& Thorpe, 1985).

Children's implicit knowledge of the hierarchical structure of Western tonal music has been examined in three studies (see Krumhansl, 1990). Krumhansl and Keil (1982) found that the performance of first and second graders reflected the influence of key membership, the performance of third and fourth graders reflected the importance of the tonic triad as well as key membership, and only adult performance showed effects of the importance of the tonic note over the other notes of the tonic triad. Speer and Meeks (1985), however, found no difference between second and fifth graders; both groups showed implicit knowledge of key membership and of the importance of the tonic triad and tonic note. Cuddy and Badertscher (1987) found that the performance of first through sixth graders revealed effects of all of these structural features, although to a lesser extent than performance by adults.

Variations in performance across the aforementioned studies are likely attributable to differences in procedure. Butler (1989) contends, however, that the relative incidence of specific notes in the stimuli of these studies could have accounted for the obtained pattern of performance (but see Cuddy, 1991). He is also critical of the use of static structures rather than melodies that unfold over time. In light of the inconsistent developmental findings and questions about the probe tone methodology, it is fair to say that the developmental course of learning about various aspects of Western tonal structure remains unclear.

In contrast to the case of melodic structure, there is litthe empirical work on the perception of implied harmony. According to Western music theory, there may be more than one appropriate harmonization of a melody. For instance, Bach sometimes harmonized the same melody in different ways in his chorale settings (e.g., Jesu, Meine Freude). Nevertheless, a simple, well-structured melody would be expected to have an obvious, or prototypical, implied harmony, although other harmonizations would be possible in principle. Indeed, good agreement is evident among music experts for simple, well-structured melodies, and variations in harmonization increase as melodies deviate from the rules of Western tonal structure (Cuddy, Cohen, \& Mewhort, 1981).
We had five musicians provide harmonic analyses of Trainor and Trehub's (1992) simple 10-note melody. Three of the musicians characterized the melody as implying a tonic chord (on notes $\mathrm{C}_{4}, \mathrm{E}_{4}$, and $\mathrm{G}_{4}$ ) followed by a dominant seventh chord (on notes $F_{4}, D_{4}$, and $G_{3}$ ), followed by a tonic chord (on notes $C_{4}, E_{4}, D_{4}$, and $C_{4}$ ). The other two musicians harmonized the melody in a very similar way, but added a dominant chord on the second to last note, yielding a tonic chord (on notes $\mathrm{C}_{4}, \mathrm{E}_{4}$, and $G_{4}$ ), followed by a dominant seventh chord (on notes $F_{4}$, $D_{4}$, and $G_{3}$ ), followed by a tonic chord (on notes $C_{4}$ and $E_{4}$ ), followed by a dominant chord (on note $D_{4}$ ), followed by a tonic chord (on note $\mathrm{C}_{4}$ ). Thus, the musicians were uniform in indicating an implied dominant harmony at the point of the potential note change (i.e., $\mathrm{G}_{3}$ ) in Trainor and Trehub's (1992) study. Furthermore, the within-key change in this study $\left(G_{3}\right.$ changed to $\left.B_{3}\right)$ also remained within the implied dominant harmony (G B D F chord) at that point. As a result, the adults' poor performance on this change may have stemmed from the harmonic function of the changed note as well as from its withinkey status.

The principal goal of Experiment 1 was to examine the contributions of key membership and implied harmony to listeners' perception of melodic changes. We used the same melody as in Trainor and Trehub (1992), as well as the same change that introduced an out-of-key note, designated out-of-key change $\left(G_{3}\right.$ was changed to $A b_{3}$ in $\mathrm{C}$ major). However, we included two within-key changes as well. One, designated within-harmony, was consistent with the implied harmony $\left(G_{3}\right.$ was changed to $B_{3}$ in $C$ major); the other, designated out-of-harmony, was not $\left(G_{3}\right.$ to $A_{3}$ in $C$ major). In all three cases, the changed note was not present in the standard melody. We hypothesized that the adults would demonstrate implicit knowledge of key membership, performing better on the out-of-key than on the within-key changes, as in previous research (Cuddy et al., 1979; Trainor \& Trehub, 1992). Adults, even those who are musically unsophisticated, have had considerable exposure to harmony in Western music, so we expected that their performance would be affected by implied harmony (as in Platt \& Racine, 1994; Thompson, 1993). Specifically, we expected them to perform better on the outof-harmony than on the within-harmony change. Never theless, the cross-cultural rarity of harmony might reflect its relative difficulty. Thus, musically untrained listeners might be less affected by implied harmony than by key membership, leading to superior performance on the outof-key change (based on key membership) over the outof-harmony change (based on implied harmony). In this case, the predicted order of difficulty for adult nonmusicians, from least to most difficult, would be out-of-key, out-of-harmony, and within-harmony. We arranged these conditions so that the use of nonmusical strategies, based on the relative magnitude of the pitch changes, would yield the opposite order: within-harmony (change of four semitones), out-of-harmony (change of two semitones), and 
out-of-key (change of one semitone). On the other hand, if exposure to Western music results in implied harmony's being an integral part of melodic perception by adulthood, then high but undifferentiated performance would be expected for out-of-key and out-of-harmony changes, because both changes involve structural deviations from the representation of the original melody.

\section{EXPERIMENT 1}

\section{Method}

Subjects. The subjects were 18 adults ( 6 male, 12 female) 17-39 years of age ( $M=23$ years), who completed all three conditions. All reported normal hearing and were free of colds at the time of testing. Three other adults were eliminated for failing to meet the training criterion. None of the adults were professional or serious amateur musicians, although some had taken music lessons (6 had taken no lessons, 7 had taken less than 8 years of lessons, and 5 had taken more than 8 years of lessons).

Apparatus. The participants were tested individually in an Industrial Acoustics Co. sound-attenuating booth. The sine-wave tones were generated by two Hewlett-Packard 3325A synthesizer/function generators, attenuated by two Med Associates attenuators, turned on and off by two Med Associates rise/fall switches, amplified by a Marantz 1070 stereo amplifier, and presented through a single Avant 2AX loudspeaker. The audio equipment, touch-sensitive response recorder, and reinforcing toys were controlled by an ECS microcomputer through a custom-made interface.

Stimuli. The standard melody (see Figure 1) was identical to that of Trainor and Trehub (1992), consisting of the notes $C_{4}, E_{4}, G_{4}$,

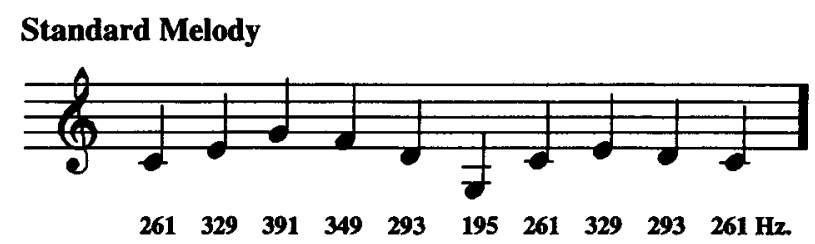

\section{Condition 1: Out-of Key Change}

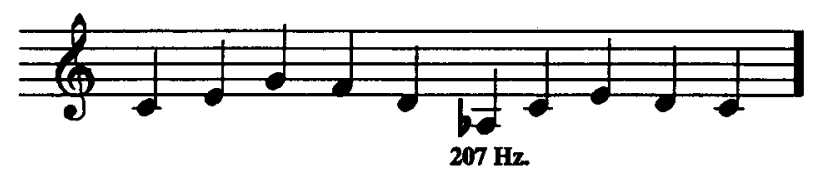

Condition 2: Out-of-Harmony Change

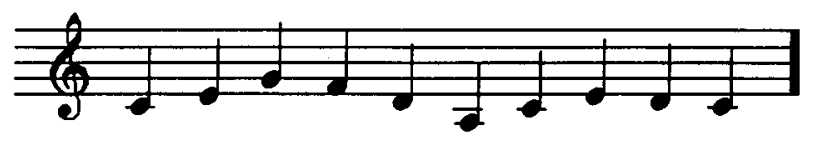

$220 \mathrm{~Hz}$.

\section{Condition 3: Within-Harmony Change}

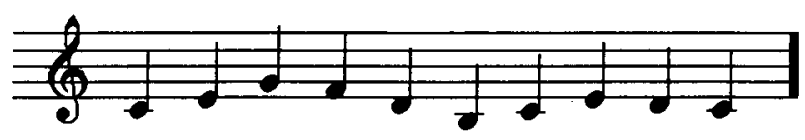

$246 \mathrm{~Hz}$.

Figure 1. The standard melody and change in the sixth note for each condition.
$F_{4}, D_{4}, G_{3}, C_{4}, E_{4}, D_{4}$, and $C_{4}(262,329,391,349,293,195,262$, 329,293 , and $262 \mathrm{~Hz}$ ) in the key of $\mathrm{C}$ major. The melody was repeated in transposition to three different keys, or starting notesspecifically, $\mathrm{C}$ major, $\mathrm{E}$ major, and $\mathrm{A} b$ major-in a quasi-random sequence so that no two consecutive melodies were in the same key. The tones were contiguous sine waves $400 \mathrm{msec}$ in duration, including 10-msec linear rise and decay times. The intermelody interval was $800 \mathrm{msec}$. The average intensity level was $75 \mathrm{~dB}(\mathrm{~A})$, and the ambient noise level was $27 \mathrm{~dB}(\mathrm{~A})$ or $42 \mathrm{~dB}(\mathrm{C})$, measured at the approximate location of the listener's head.

The standard melody was typical of Western tonality in the sense that all the notes belonged to one key, and it had a simple implied harmony: the first three notes implied a tonic harmony (C E G), the next three a dominant harmony (G B D F), and the final four either a tonic harmony or a tonic-dominant-tonic harmony. The listener's task in each of the three conditions was to detect a change in the sixth note of the standard melody (see Figure 1). In the outof-key condition, $\mathrm{G}_{3}$ (in the $\mathrm{C}$ major rendition of the melody) was raised one semitone to $A b_{3}(207 \mathrm{~Hz})$, a note outside the key. In the out-of-harmony condition, $G_{3}$ was raised two semitones to $A_{3}$ $(220 \mathrm{~Hz})$, a note within the key of $\mathrm{C}$ major, but outside the implied dominant harmony. In the within-harmony condition, $G_{3}$ was raised four semitones to $B_{3}(246 \mathrm{~Hz})$, a note within the key as well as within the prevailing implied harmony.

The changes in the training phase (see Procedure below) were larger and of the same magnitude for all conditions. In the first three training trials, $\mathrm{G}_{3}$ was lowered six semitones to $\mathrm{CH}_{3}(139 \mathrm{~Hz})$; in the remaining training trials, it was lowered four semitones to $\mathrm{E}_{b_{3}}(156 \mathrm{~Hz})$.

Procedure. We used the procedure of Trainor and Trehub (1992) to enhance comparability. The listeners were seated in the soundattenuating booth opposite the experimenter, who wore headphones and listened to masking music so as to be unaware of whether a control or change trial was being presented. The standard melody was repeated continuously in transposition in a quasi-random order, so that no two consecutive repetitions were at the same pitch level throughout the test session. The repeating standard melody constituted the background against which the detection of changes was evaluated. When the participant was attentive, the experimenter called for a trial by pressing a button on the response recorder. Trials were not identified in any way, for either the experimenter or the listener. There were variable numbers of repetitions of the standard melody between trials; the minimum was two. On half the trials, the melody was presented with the changed note (see Stimuli); on the other half, or control trials, repetitions of the standard melody simply continued. Change and control trials were presented in a quasi-random order (no more than three consecutive control trials), for a total of 24 trials per condition. The listeners were instructed to raise a hand when they detected a change. If they responded correctly during the 3-sec period that began with the sixth (changed) note of the melody, an animated toy was activated and illuminated for $4 \mathrm{sec}$, as in the infants' procedure of Trainor and Trehub (1992). No feedback was provided for responses at other times. The computer recorded all responses during this 3 -sec response interval on both change and control trials.

The test phase of each condition was preceded by a training phase with larger changes (see Stimuli). Failure to respond to a change led to the presentation of the next change melody at a level that was $5 \mathrm{~dB}$ greater than that of the background melody. Correct responding resulted in a $5-\mathrm{dB}$ decrease in the intensity of the next change melody until background and change melodies were equivalent, as they remained for the subsequent test phase. Participants who did not meet a training criterion of four consecutive correct responses (at equivalent background and change intensity) within 20 trials were eliminated. Each participant completed all three conditions in a single test session of approximately $45 \mathrm{~min}$. There were 3 participants in each of the six possible orders of the three conditions. 


\section{Results and Discussion}

For each participant in each condition, the proportions of responses on change trials (hits) and control trials (false alarms) were transformed to $d^{\prime}$ scores according to yes/no tables of signal detection theory (Elliot, 1964). Occasional proportions of 0 or 1 present a problem because they result in infinite $d^{\prime}$ scores. These scores are believed to be statistically infinite rather than truly infinite (see Macmillan \& Kaplan, 1985), arising from the sampling error inherent in a limited number of trials. To circumvent this difficulty, we transformed the scores prior to conversion to $d^{\prime}$. Proportions were calculated by adding $1 / 2$ to the number of actual responses (out of 12) and dividing by the number of possible responses plus 1 (i.e., 13). This transformation maintains the original ranking of scores and has little effect on $d^{\prime}$ values (see Thorpe, Trehub, Morrongiello, \& Bull, 1988). A $d^{\prime}$ of 0 represents chance performance; the maximum $d^{\prime}$ achievable under these conditions is 3.50 .

A repeated measures analysis of variance (ANOVA) with type of change and order of conditions as factors revealed no effect of order and no interaction between order and change, although power was low with only 3 subjects per order. There was, however, a significant effect of change $[F(2,24)=14.34, p<.0001$; see Figure 2]. Paired-samples $t$ tests, with the Bonferroni correction for multiple tests, revealed that performance on the out-of-key and out-of-harmony changes (mean $d$ 's of 2.64 and 2.69, respectively) did not differ. Furthermore, performance on the within-harmony change (mean $d^{\prime}$ of 1.80 ) was significantly lower than performance on the other conditions $[t(17)=4.07, p<.003$ for out-of-key vs. withinharmony; $t(17)=5.36, p<.002$ for out-of-harmony vs. within-harmony].

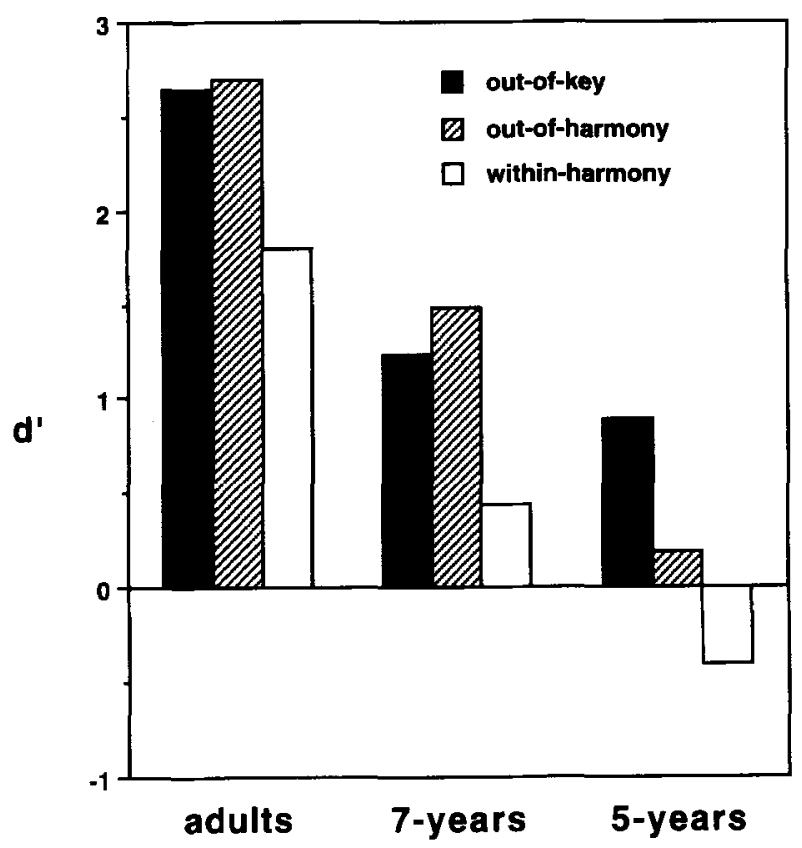

Figure 2. Performance across age and type of change.
These results are consistent with the interpretation that adults have implicit knowledge of both key membership and implied harmony. The adults performed significantly better on the out-of-harmony than on the within-harmony change, confirming the role of implied harmony in the perception of tone sequences. Their high level of performance on the out-of-key change suggests implicit knowledge of key membership, although ceiling effects precluded testing for differences between performance on the out-of-key and out-of-harmony conditions.

Musical training (i.e., number of years of music lessons) was significantly correlated with performance on the within-harmony $[r=.53, t(16)=6.08, p<.03]$ and out-of-harmony $[r=.60, t(16)=2.98, p<.01]$ changes, reflecting the progressive refinement of analytical listening skills over the course of formal training. By contrast, musical training was uncorrelated with performance on out-of-key changes $(r=.27)$, indicating that knowledge about key membership can be acquired by simple exposure.

The adults' high level of performance on the out-ofharmony change indicates their internalization of the principles of implied harmony. Years of informal exposure to Western music likely contributes to adults' knowledge of implied harmony. However, the presence of a substantial correlation between training and performance on the within-key changes and its absence on the out-of-key change provide suggestive evidence that more learning is required for implied harmony than for key membership. Thus, listeners with considerably less exposure to Western music might exhibit knowledge of key membership, but not implied harmony.

\section{EXPERIMENT 2}

Musical systems are typically based on a small number of discrete pitches per octave, although the specific pitches may differ across cultures. Thus, listeners, regardless of culture, must learn the culture-specific sets of pitches. By contrast, the Western prominence of harmonic function is atypical in cross-cultural perspective, obviating most listeners' need to learn this aspect of musical structure. In Experiment 1 we demonstrated, however, that Western nonmusicians encode melodies in terms of their implied harmonies, even in the absence of simultaneously sounded tones. Thus, extended exposure to Western music is sufficient for the acquisition of implicit knowledge of key membership and implied harmony, both of which are clearly absent in infancy (Trainor \& Trehub, 1992). On the basis of key membership as a universal, or nearuniversal, musical function and implied harmony as a relatively rare function, one would expect information about key membership to be acquired earlier and more readily than information about implied harmonic function.

In Experiment 2, we assessed 5- and 7-year-old children's implicit knowledge of key membership and implied harmonic function. We used the same melody and changes that were used in Experiment 1, but made some procedural changes to accommodate children's response ca- 
pabilities. First, the melodies were not presented in transposition, because pilot testing revealed that children respond to the transpositional change as well as to the relative pitch changes of interest. Second, discrete trials were presented instead of the continuous repetitions of Experiment 1 , because of children's propensity to initiate conversations from time to time, thereby obscuring the auditory stimuli. Thus, discrete nontransposed trials allowed for superior management of children's attention. Third, we used a between-subjects design because of the difficulty of maintaining children's interest in three successive sessions.

\section{Method}

Subjects. The subjects were forty-two 5-year-olds (range = 4 years, 9 months to 5 years, 3 months; $M=5$ years, 26 days) and forty-two 7 -year-olds (range $=6$ years, 9 months to 7 years, 3 months; $M=7$ years, 10 days). There were 20 boys and 22 girls in the younger group and 15 boys and 27 girls in the older group; all had normal hearing (according to parental report) and were free of colds at the time of testing. An additional twenty-one 5-year-olds and four 7-year-olds were excluded from the sample for three reasons: failure to meet the training criterion (5-year-olds: $n=11$; 7-year-olds: $n=3$ ); inadequate attention (5-year-olds: $n=4$; 7-year-olds: $n=1$ ), as indicated by incorrect responding on more than two out of three probe trials (see Procedure); or failure to complete the entire test session (5-year-olds: $n=6$ ). Most of the children had had no formal musical training (5-year-olds: 2 had 1 year of piano lessons; 7 -year-olds: 2 had 1 year and 2 had 2 years of piano lessons).

Stimuli. The melodies and changes were identical to those of Experiment 1 , except that all melodies were presented in the key of $\mathrm{C}$ major.

Apparatus. The apparatus was identical to that of Experiment 1.

Procedure. The children participated in a same-different task. On each trial, two melodies were presented-the first was the standard melody and the second was the comparison melody. On change trials ( $n=10$ ), the second melody incorporated the change for that condition (out-of-key, out-of-harmony, or within-harmony; see Stimuli); on control trials $(n=10)$, the second melody was identical to the standard. The experimenter, who was blind to the hypotheses of the study, sat in the sound-attenuating chamber across from the child. The children were first trained in the use of the terms "same" and "different" with colored blocks. They were presented with two identical blue blocks and asked if they were the same or different. Subsequently, they received two trials with a blue and green block of different size and were asked if these were the same or different. Finally, they were presented with two red blocks of different size. When it seemed clear that the children understood the appropriate application of "same" and "different," the experimenter proceeded to the task of interest.

The children were told to listen to a "special song" and were given three presentations of the standard melody. Subsequently, they were told that they would hear the special song (i.e., standard melody) followed by another song that might be exactly the same as the special song, or a little different. They could indicate whether it was the same or different either verbally or by pressing one of two buttons (negotiated individually at the start of the training phase). There were two identical pictures of a cat beside the same button; a picture of a cat and another of a dog were beside the different button. The experimenter recorded the child's responses on the response recorder, which was linked to the computer. Correct responding on same and different trials resulted in the activation of a light and toy for $4 \mathrm{sec}$. Prior to the test trials, the children received training trials in which the to-be-detected change was much larger (see Stimuli). The children were required to produce four consecutive correct responses (including at least one same and one different trial) within 20 trials before proceeding to the test trials. During the test phase, there were three probe trials in which the comparison (second) melody incorporated the training change; each probe trial occurred after every five test trials. These probe trials were used as a measure of attention; the children who failed to detect the change on at least two of the three probe trials were excluded from the final sample (see Subjects), which consisted of 14 children for each age group in each of the three conditions.

\section{Results and Discussion}

Proportions of hits and false alarms were calculated and transformed to $d^{\prime}$ scores, according to yes/no tables, as in Experiment 1. Figure 2 shows mean $d^{\prime}$ 's for each condition across age levels. ANOVAs with type of change (out-of-key, out-of-harmony, within-harmony) as a factor were performed separately for the 5- and 7-year-olds. In both cases, there was a significant effect of type of change $[F(2,39)=15.90, p<.0001$ for the 5-year-olds; $F(2,39)=3.44, p<.05$ for the 7 -year-olds]. However, Tukey's $H S D$ multiple comparisons revealed different patterns of performance for the two age groups. The 7-yearolds showed a pattern of performance that was similar to the adults', with statistically equivalent performance on the out-of-key (mean $d^{\prime}$ of 1.23) and out-of-harmony (mean $d^{\prime}$ of 1.48) changes and superior performance on the out-of-key change over the within-harmony change (mean $d^{\prime}$ of 0.44 ). By contrast, the 5-year-olds performed better on the out-of-key change (mean $d^{\prime}$ of 0.88 ) than on the other two types of changes $\left(d^{\prime}=0.19\right.$ on the outof-harmony; $d^{\prime}=-0.41$ on the within-harmony), which were not above chance levels.

Performance on the within-harmony change was significantly below chance levels $[t(13)=2.23, p<.05]$ for the 5-year-olds. We do not have a definitive interpretation of this result. From the perspective of prototype theory (see, e.g., Trehub \& Unyk, 1991), however, the within-harmony melody (containing the $\mathrm{B}_{3}$ ) may be a "better" or more typical melody than the standard melody (containing the $\mathrm{G}_{3}$ ). According to Narmour (1990), innate expectations about interval size and direction lead listeners to expect a small downward interval (such as that between the fourth and fifth notes-three semitones) to be followed by another small downward interval. This expectation would be fulfilled for the within-harmony melody (with a three-semitone interval between the fifth and sixth notes), but not for the standard melody (with a sevensemitone interval between the fifth and sixth notes). In other words, the standard melody may be less well structured, or stable, than the within-harmony melody. At the same time, the two melodies are very similar, perhaps leading the children to mistake the more stable withinharmony melody for the standard melody-hence the below-chance performance.

In any case, the principal finding of interest was that the 5-year-olds performed better on the out-of-key change than on the other two changes, which were not above chance levels, and that the 7-year-olds performed better 
on the out-of-harmony change than on the within-harmony change. This pattern of results is consistent with the interpretation that 5-year-olds have implicit knowledge of key membership but not of implied harmony, whereas 7-year-olds, like adults, have implicit knowledge of both aspects of musical structure.

\section{GENERAL DISCUSSION}

In the present investigation, we were concerned with the role of key membership and implied harmony in adults' and children's perception of tone sequences. We expected earlier perceptual effects for key membership, which is relevant to virtually all musical systems, and later effects for implied harmony, which is relatively rare crossculturally. Presumably, different developmental timetables for these features would arise from differential ease of acquisition or from differential exposure. Our predictions were borne out: The performance of the 5-year-old children was affected by key membership but not by implied harmony, whereas that of the 7-year-old children and adults was affected by both key membership and implied harmony.

These findings together with earlier research indicate a clear developmental progression, beginning with effects of perfect fifth and octave intervals in the first year of life (Demany \& Armand, 1984; Trainor \& Trehub, 1993a, 1993b), and followed by effects of key membership by 5 years of age and implied harmony by 7 years of age. Implicit knowledge about key membership, although absent in 8-month-olds (Trainor \& Trehub, 1992), is likely to be present well before 5 years of age, which is the youngest age that has been examined postinfancy. In any case, the acquisition of implicit knowledge about implied harmony occurs rather late in development, compared with other aspects of musical structure.

What are the implications of the present developmental findings for theories of music acquisition? Krumhansl (1990, pp. 285-286) proposed that the acquisition of implicit knowledge about music structure is based primarily on the statistical properties of music. For example, the notes of the tonic chord are believed to assume perceptual prominence because they occur with greater frequency than the other notes of the scale. Extending this line of reasoning to a developmental context, children should learn about key membership because of the frequent cooccurrence of certain notes (i.e., notes of a scale) and the infrequent co-occurrence of others (i.e., nonscale with scale notes). Similarly, children should learn about implied harmony by associating melody notes with their accompanying chords. One problem with this formulation, however, is its failure to explain the developmental precedence of key membership over implied harmony. Indeed, many common children's songs (e.g., Twinkle Twinkle Little Star) do not contain all the notes of the scale. Moreover, children frequently hear recorded versions of such songs with harmonic accompaniment (e.g., TV shows like "Sesame Street" and “The Elephant Show'). Thus, although relative frequency of occurrence may influence the acquisition of specific musical structures, it seems unlikely that it can account for the present developmental findings.

The acquisition of speech sounds reveals some dissociations between statistical properties of phonemes within a language and their order of acquisition. On the whole, sounds that are universal or near-universal (e.g., ma/pa) are acquired earlier than sounds that occur in relatively few languages (e.g., la/ra) (Jakobson, 1968; Locke, 1983), even though sounds that are cross-culturally infrequent may have a high frequency of occurrence in a particular language (e.g., in English, sounds written as $t h)$. In music, as well, near-universal musical features may be acquired earlier and more easily than unique features.

\section{REFERENCES}

Aldwell, E., \& Schachter, C. (1989). Harmony and voice leading (2nd ed.). San Diego: Harcourt Brace Jovanovich.

BARTLETT, J. C., \& Dowling, W. J. (1980). Recognition of transposed melodies: A key distance effect in developmental perspective. Journal of Experimental Psychology: Human Perception \& Performance, 6, 501-515.

Bharucha, J. J. (1984). Anchoring effects in music: The resolution of dissonance. Cognitive Psychology, 16, 485-518.

Butler, D. (1989). Describing the perception of tonality in music: A critique of the tonal hierarchy theory and proposal for a theory of intervallic rivalry. Music Perception, 6, 219-242.

Cohen, A. J., Thorpe, L. A., \& Trehub, S. E. (1987). Infants' perception of musical relations in short transposed tone sequences. Canadian Journal of Psychology, 41, 33-47.

CudDy, L. L. (1991). Melodic patterns and tonal structure: Converging evidence. Psychomusicology, 10, 107-126.

CUDDY, L. L., \& BADERTSCHER, B. (1987). Recovery of the tonal hierarchy: Some comparisons across age and levels of musical experience. Perception \& Psychophysics, 41, 609-620.

Cuddy, L. L., Cohen, A. J., \& Mewhort, D. J. K. (1981). Perception of structure in short melodic sequences. Journal of Experimental Psychology: Human Perception \& Performance, 7, 869-883.

Cuddy, L. L., Cohen, A. J., \& Miller, J. (1979). Melody recognition: The experimental application of musical rules. Canadian Journal of Psychology, 33, 148-156.

Demany, L., \& ARMAND, F. (1984). The perceptual reality of tone chroma in early infancy. Journal of the Acoustical Society of America, 76, 57-66.

Deutsch, D., \& Feroe, J. (1981). The internal representation of pitch sequences in tonal music. Psychological Review, 88, 503-522.

Dowling, W. J., \& HARWOOD, D. L. (1986). Music cognition. Orlando, FL: Academic Press.

Elliot, P. B. (1964). Tables of $d^{\prime}$. In J. A. Swets (Ed.), Signal detection and recognition by human observers (pp. 651-684). New York: Wiley.

HARWOOD, D. L. (1976). Universals in music: A perspective from cognitive psychology. Ethnomusicology, 20, 521-534.

Howell, P., Cross, I., \& West, R. (Eds.) (1985). Musical structure and cognition. London: Academic Press.

JAKOBSON, R. (1968). Child language, aphasia and phonological universals. The Hague: Mouton.

KrumhansL, C. L. (1990). Cognitive foundations of musical pitch. New York: Oxford University Press.

KrumhansL, C. L., \& KeIL, F. C. (1982). Acquisition of the hierarchy of tonal functions in music. Memory \& Cognition, 10, 243-251.

LOCKE, J. L. (1983). Phonological acquisition and change. New York: Academic Press

LYNCH, M. P., \& ElLERs, R. E. (1991), Children's perception of native and nonnative musical scales. Music Perception, 9, 121-132.

LynCh, M. P., Eilers, R. E., Oller, D. K., \& Urbano, R. C. (1990). Innateness, experience, and music perception. Psychological Science, 1, 272-276. 
LynCh, M. P., Eilers, R. E., Oller, D. K., Urbano, R. C., \& WilSON, P. (1992). Influences of acculturation and musical sophistication on perception of musical interval patterns. Journal of Experimental Psychology: Human Perception \& Performance, 17, 967-975.

Macmillan, N. A., \& Kaplan, H. L. (1985). Detection theory analysis of group data: Estimating sensitivity from average hit and false-alarm rates. Psychological Bulletin, 98, 185-199.

Narmour, E. G. (1990). Analysis and cognition of basic melodic structure: The implication-realization model. Chicago: University of Chicago Press.

PICK, A. D. (1979). Listening to melodies: Perceiving events. In A. D. Pick (Ed.), Perception and its development (pp. 145-165). New York: Wiley.

Platt, J. R., \& Racine, R. J. (1994). Detection of implied harmony changes in triadic melodies. Music Perception, 11, 243-264.

SHEPARD, R. N. (1982). Geometrical approximations to the structure of musical pitch. Psychological Review, 89, 305-333.

Sloboda, J. A. (1985). The musical mind: The cognitive psychology of music. Oxford: Clarendon Press.

SPEER, J. R., \& MEEKs, P. U. (1985). School children's perception of pitch in music. Psychomusicology, 5, 49-56.

TAKeUChI, A. H., \& HulSE, S. H. (1992). Key-distance effects in melody recognition reexamined. Music Perception, 10, 1-24.

TERHARDT, E. (1991). Music perception and sensory information acquisition: Relationships and low-level analogies. Music Perception, 8, 217-240.

ThOMPSon, W. F. (1993). Modeling perceived relationships between melody, harmony, and key. Perception \& Psychophysics, 53, 13-24.

Thompson, W. F., \& CUdDY, L. L. (1989). Sensitivity to key change in chorale sequences: A comparison of single voices and four-voice harmony. Music Perception, 7, 151-168.

Thompson, W. F., \& Cuddy, L. L. (1992). Perceived key movement in four-voice harmony and single voices. Music Perception, 9 , 427-438.

Thorpe, L. A., Trehub, S. E., Morrongiello, B. A., \& Bull, D. (1988). Perceptual grouping by infants and preschool children. Developmental Psychology, 24, 484-491.

Trainor, L. J., \& Trehub, S. E. (1992). A comparison of infants' and adults' sensitivity to Western musical structure. Journal of Experimental Psychology: Human Perception \& Performance, 18, 394-402.
Trainor, L. J., \& Trehub, S. E. (1993a). Musical context effects in infants and adults: Key distance. Joumal of Experimental Psychology: Human Perception \& Performance, 19, 1-13.

Trainor, L. J., \& Trehub, S. E. (1993b). What mediates infants' and adults' superior processing of the major over the augmented triad? Music Perception, 11, 185-196.

TrehUb, S. E., Bull, D., \& ThorPe, L. A. (1984). Infants' perception of melodies: The role of melodic contour. Child Development, 55, 821-830.

Trehub, S. E., Morrongiello, B. A., \& Thorpe, L.A. (1985). Children's perception of familiar melodies: The role of intervals, contour, and key. Psychomusicology, 5, 39-48.

Trehub, S. E., Thorpe, L. A., \& Morrongiello, B. A. (1985). Infants' perception of melodies: Changes in a single tone. Infant Behavior \& Development, 8, 213-223.

Trehub, S. E., Thorpe, L. A., \& Morrongiello, B. A. (1987). Organizational processes in infants' perception of auditory patterns. Child Development, 58, 741-749.

Trehub, S. E., ThorPe, L. A., \& Trainor, L. J. (1990). Infants' perception of good and bad melodies. Psychomusicology, 9, 5-15.

Trehub, S. E., \& Trainor, L. J. (1990). Rules for listening in infancy. In J. Enns (Ed.), The development of attention: Research and theory (pp. 87-119). Amsterdam: Elsevier.

TrEhub, S. E., \& Trainor, L. J. (1993). Listening strategies in infancy: The roots of language and musical development. In S. McAdams \& E. Bigand (Eds.), Thinking in sound: The cognitive psychology of human audition (pp. 278-327). London: Oxford University Press.

Trehub, S. E., Trainor, L. J., \& UNYK, A. M. (1993). Music and speech processing in the first year of life. Advances in Child Behavior \& Development, 24, 1-35.

Trehub, S. E., \& UNYK, A. M. (1991). Music prototypes in developmental perspective. Psychomusicology, 10, 31-45.

(Manuscript received July 8, 1993; revision accepted for publication December 31, 1993.) 